\title{
The Application of Chomsky's Syntactic Theory in Translation Study
}

\author{
Ying $\mathrm{Wu}$ \\ Zhenjiang Watercraft College of PLA, Zhenjiang, Jiangsu, 212003, China \\ Email: wycherry@ sina.com \\ Runjiang Xu \\ Zhenjiang Watercraft College of PLA, Zhenjiang, Jiangsu, 212003, China
}

\begin{abstract}
Noam Chomsky is a famous American linguist. Since he put forward his Transformationalgenerative Grammar (TG), many scholars have tried to use the concepts of deep structure (D-structure) and surface structure (S-structure) to provide a theoretical basis for translation studies. It is true that the theory of $\mathrm{D}$ - / S-structure is of great importance in analyzing the syntactic structure of sentences in source language and target language. However, in the author's opinion, the function of D- / S-structure cannot be exaggerated in translation study as translation is not just a syntactic process but a combination of many aspects.
\end{abstract}

Index Terms - Chomsky, Syntactic Theory, translation

\section{CHOMSKY AND His SYNTACTIC THEORY}

Chomsky is one of the most popular linguists in modern linguistic studies. His Transformational-generative Grammar (TG) has a revolutionary influence in this field since he published his first book Syntactic Structures in 1957(Chomsky, 1957). In his theory, Chomsky points out that there are two levels of syntactic structure in every sentence. The first is called deep structure or D-structure, which is formed by the XP rule. Deep structure plays an important role in the interpretation of sentences. The second is called surface structure or S-structure, which is formed by applying appropriate transformations for the deep structure of sentence in question. The following figure may explain clearly the relationship between them:

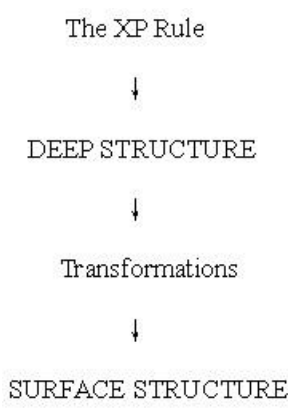

Figure 1 Deep Structure and Surface Structure

The XP rules generate an underlying deep structure which is transformed by transformational rules to produce a final surface structure. The surface structure itself is subject to phonological and morphemic rules. We can use tree diagram to analyze the organization and relationship of different components of a sentence. To some structurally ambiguous sentences, two or more deep structures are possible for one surface structure (Gong Xiaobin, 2004).

\section{INFLUENCE OF CHOMSKY'S THEORY ON TRANSLATION THEORY}

Generally speaking, translation is an activity of expressing something in another language without changing its original meaning. The greatest problem in translation study is the lack of a systematic theory to direct the translation activities. A lot of discussions have been around whether translation is a science or not. If it is a science, then what is the general principle of translation? What is the standard to distinguish good and bad translations? Translators and theorists have long been working for establishing a scientific basis for translation studies. They made great effort from different angles. Linguistics is where they have paid most attention as both linguistics and translations are the study of language. Therefore, as soon as Chomsky's TG theory came into being, scholars have been interested in applying this theory to the scientific research of translation. American translator Eugene Nida is one of the most famous figures in this attempt.

Nida's theory took form in two major works in the 1960s. The first is Toward a Science of Translating published in 1964 (Nida, 1964). The second is The Theory and Practice of Translation, co-authored with Taber in 1969 (Nida and 
Taber, 1969). In his theory, Nida incorporates key features of Chomsky's model into his 'science' of translation. He sees that it provides the translator with a technique to decode the Source Text (ST) and a procedure to encode the Target Text (TT). Thus, the surface structure of the ST is analyzed into the basic elements of the deep structure. These are 'transferred' in the translation process and then restructured semantically and stylistically into the surface structure of the TT (Nida and Taber, 1969). This three-stage system of translation (analysis, transfer and restructuring) is presented in the following figure.

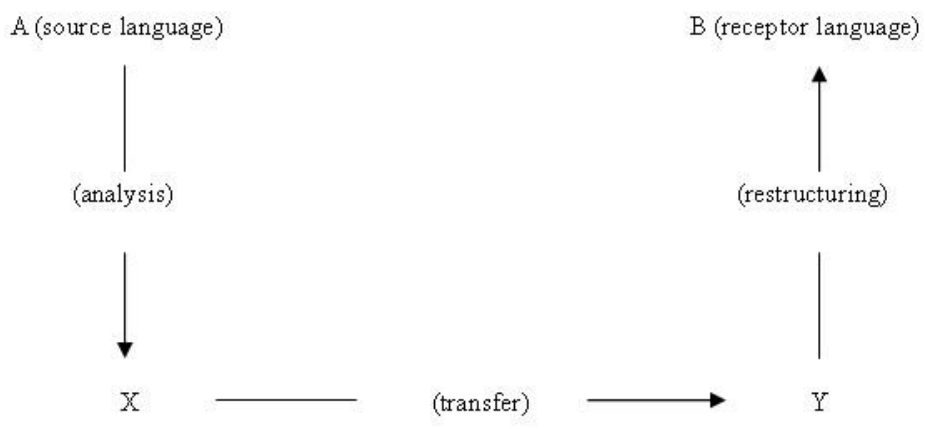

Figure 2 Nida's Translation Model

It can be seen clearly from the figure that Nida reverses Chomsky's model in the stage of analysis. As Chomsky focuses on the generation of a surface structure through transformation, Nida aims at analyzing the ST surface structure by a reductive process of back-transformation. 'Kernel' is a key term in this model just as kernel sentences were the most basic structural elements in the early works of Chomsky. Kernels are obtained from the ST surface structure by back-transformation analysis. They are the basic elements in deep structure. This involves the four types of functional class in transformational-generative grammar (Jeremy, 2001).

- events (often but not always performed by verbs);

- objects (often but not always performed by nouns);

- abstracts (quantities and qualities, including adjectives);

- relationals (including gender, prepositions and conjunctions).

Nida claims that all languages have about six to a dozen basic kernel structures and they agree more on the level of kernels than on the level of more elaborate structures. Nida's statement is actually under the influence of Chomsky's Universal Grammar (UG), which believes that all human languages have a shared system of categories, mechanisms and constraints. It needs to be clarified that UG is not a grammar system that suits every human language, but the mechanism or architecture of all grammars. In a word, it's the grammar of grammars. If the existence of UG can be justified, then the translatability between different languages can be proved scientifically. That is to say, all languages can be transferred in their deep structures because they share the 'kernels'.

\section{LIMITATION OF THE SYNTACTIC THEORY IN TRANSLATION}

Just as we have mentioned, Chomsky's syntactic theory may help to prove the translatability between human languages. However, in the author's opinion, TG theory alone is not enough to prove that translation as a whole is a science. TG theory or D- / S-structure theory in particular is mainly on the study of syntax. It's true that they help a lot in sentence analysis, especially for some complicated and structurally ambiguous sentences, but many other translation problems cannot be solved only with TG theory.

\section{A. Syntax and Sentence Meaning}

Some scholars believe that deep structure is the source of sentence meaning. Some even say that deep structure determines the meaning of sentence and surface structure is the phonological and morphemic expression of deep structure (Lefevere, 1993). However, in the author's opinion, TG theory is mainly on the analysis of sentence structure. It focuses on the rules of the generation and transformation of sentences. Deep structure as a syntactic concept can only determine part of the sentence meaning, the part that is caused by the organization of words or phrases, for example, the subject, object etc. As sentence meaning is also determined by the context, culture or even some psychological factors of speaker, it should not be equaled with the deep structure (van den Broeck, 1978: 40). As for translation study, it's impractical to only depend on kernels in deep structure to transfer the meaning of a sentence. For example, in translating idioms or set phrases, the analysis of deep structure may be helpless. The following example is about translating an English idiom into Chinese.

ST: Someone will have to break the ice.

TT1: 有人一定会把冰敲破的。

TT2: 总得有人先开口说话。

(ST: Source Text, TT: Target Text) 
The deep structure of the source text is not difficult to analyze. 'break the ice' is a verbal phrase, and 'ice' is the direct object of verb 'break'. However, does the syntactic analysis provide enough information for translation? Definitely not. If we are English beginners and have little background knowledge of English idioms or cultures, we will most likely translate it as TT1. If we know that 'break the ice' in most cases is a set phrase and means 'break the silence' or 'start to talk', we will certainly make a different translation like TT2. Of course, as we have no context here, we cannot decide which translation, TT1 or TT2, is the correct one. On some special occasion, 'break the ice' may be used as a pun to have both meanings. Anyway, this example is enough to illustrate that syntactic analysis alone is not enough to determine the sentence meaning. Therefore, it's cannot act as the only information source of translation.

\section{B. TG Theory is Insufficient to Prove the Scientificity of Translation}

As have been mentioned in part 2, scholars in translation study employ Chomsky's TG theory to try to provide a scientific basis for translation. Eugene Nida uses kernels as the minimum translation units and has proved the translatability between different languages. However, in the author's view, it's far from saying that translation is a science, or translation activity can be conducted in a scientific way. To be a science, it needs at least an objective standard or principle to judge what is right and what is wrong. If according to some scholars, deep structure can serve as the principle, then how can we explain problems in the following pair of sentences?

ST1: She sings beautifully.

TT1:她唱得很美。

ST2: Her singing is beautiful.

TT2: 她的歌声很美。

There seems to be no problem if ST1 is translated to TT1 and ST2 to TT2. According to TG theory, it is clear that ST1 and ST2 have different deep structures because they have different syntactic relationships. Therefore, according to Nida's theory, they should have different deep structures in Chinese respectively, and TT1 is undoubtedly a correct translation of ST1 and TT2 of ST2. However, if we make a small change and translate ST1 into TT2 or ST2 into TT1, will there be a mistake? With common sense, we may find it acceptable as well, or at least, it makes sense. We can hardly say it is a wrong translation. Then, if it is also a correct translation, it means one deep structure in one language may correspond to two or even more different deep structures in another language. How can this be a science?

Somebody may argue that it is because ST1 and ST2 have the same meaning in their deep structures. Then there comes two different deep structures with the same meaning, and we will come back to our discussion in 3.1, that is deep structure or syntax is not the only decisive factor of sentence meaning, or it cannot contain all the factors concerned with meaning. As for translation, TG theory on syntax is not sufficient to serve as the objective principle to distinguish the correct from the wrong. Therefore, the scientificity of translation study is still need to be proved.

\section{PRACTICAL APPlication OF TG THEORY IN TRANSLATION}

Although presently it is hard to say translation is a science, it doesn't mean there is nothing scientific in translation activity or the application of TG theory in translation study is meaningless. Nowadays, TG theory and some other methods such as corpus technique are widely used in machine translation. Researches are making great effort to imitate the translation process of human being in computers. Though not sufficient to solve all the problems in translation, TG theory and Nida's model are practical in most routine translation activities. To understand it better, it is necessary to distinguish literature translation from non-literature translation in our translation studies. According to Nida (1969), literature translation only accounts for no more than 5\% of all translation activities. Compared with literature translation, non-literature translation covers much more fields from politics, economics, legislation to science, technology and many other areas. It has less aesthetic requirement and is more systematic in sentence structure than literature translation. For some less strict non-literature translation tasks, computer may help us a lot and save our time and energy to a great extent. Undoubtedly, the development in linguistics especially in TG theory will provide powerful support for the development of machine translation.

\section{CONCLUSION}

The translation study is by its nature an interdisciplinary subject. It concerns knowledge of languages, linguistics, communication studies, philosophy and a range of types of cultural studies. The developing history of translation studies is actually a process of looking for a systematic theory for translation activities. However, the diversity in its nature determines the difficulty of this process. From generation to generation, great efforts have been made from different aspects of translation, especially from linguistics. The influence of Chomsky's TG theory to Nida's translation model is a good example of this combination. Of course, as we have analyzed, many translation problems cannot be solved in this model yet, but I'm sure the development in translation studies will soon give us a better answer.

\section{REFERENCES}

[1] Broeck, R. van den (1978). 'The concept of equivalence in translation theory: Some critical reflections', in J. S. Holmes, J. Lambert and R. van den Broeck (eds) Literature and Translation, Leuven: Academic, 29-47. 
[2] Chomsky, N. (1957). Syntactic Structures. The Hague: Mouton.

[3] Gong Xiaobin (2004). A Cross-Discipline Study of Surface Structure and Deep Structure. Journal of Social Science of Hunan Normal University 33, 23-25.

[4] Jeremy Munday. (2001). Introducing Translation Studies theories and applications. New York: Routledge,

[5] Lefevere, A. (1993). Translating Literature: Practice and Theory in a Comparative Literature Context. New York: The Modern Language Association of America.

[6] Nida, E. (1964). Towards a Science of Translating. Leiden: E.J. Brill.

[7] Nida, E and C, Taber. (1969). The Theory and Practice of Translation. Leiden: E.J. Brill.

Ying Wu was born in Zhenjiang, China in 1979. She received his M.A. degree in Translation Study from Nanjing University, China in 2009.

She is currently a lecturer in the English Department of Zhenjiang Watercraft College of PLA, Zhenjiang, China. Her research interests include Linguistics and Foreign Language Teaching.

Runjiang Xu was born in Zhenjiang, China in 1984. She receives her M.A. degree in Foreign Linguistics and Applied Linguistics from Jiangsu University, China in 2010.

She is currently a lecturer in English Department, Zhenjiang Watercraft College of PLA, Zhenjiang, China. Her research interests include Second Language Acquisition and Foreign Language Teaching. 